\title{
Sound attenuation in the superconducting amorphous alloy ZrTiCuNiBe
}

\author{
E. V. Bezuglyı̆, A. L. Gaiduk, and V. D. Fil \\ B. Verkin Institute for Low Temperature Physics and Engineering, National Academy of Sciences of the \\ Ukraine, 310164 Kharkov, Ukraine*
}

W. L. Johnson

California Institute of Technology, Pasadena, CA 91125, USA

G. Bruls, B. Lüthi, B. Wolf, and S. V. Zherlitsyn

Physikalisches Institut, Universitat Frankfurt, 60054 Frankfurt, Germany

(Submitted May 25, 1999)

Fiz. Nizk. Temp. 25, 1329-1333 (December 1999)

The superconducting energy gap and the parameter $\eta$ determining the intensity of electron scattering at two-level systems in amorphous $\mathrm{ZrTiCuNiBe}$ are determined from the results of measurements of sound attenuation. The mechanism of adiabatic renormalization of the amplitude of coherent tunneling is used for a quantitative description of the peculiarities of sound absorption in the vicinity of $T_{c}$. (C) 1999 American Institute of Physics.

[S1063-777X(99)01312-2]

Preliminary measurements of the velocity $v$ and absorption $\Gamma$ of sound in the amorphous alloy $\mathrm{Zr}_{1.2} \mathrm{Ti}_{13.8} \mathrm{Cu}_{12.5} \mathrm{Ni}_{10} \mathrm{Be}_{22.5}$ have revealed ${ }^{1}$ an interesting peculiarity in its behavior in the vicinity of the superconducting transition temperature $T_{c}$. It was found that the value of $T_{c m} \approx 0.9 \mathrm{~K}$ determined from magnetic measurements exceeds the transition temperature $T_{c} \approx 0.83 \mathrm{~K}$ at which a nonzero difference is observed in the velocities of sound in superconducting $(s)$ and normal $(n)$ phases. It was proposed that such a behavior may be due to magnetic depairing leading to the gapless $s$-phase in the temperature interval $T_{c m}-T_{c}$. Since our earlier measurements ${ }^{1}$ were made in a temperature interval limited from below $(T \gtrsim 0.4 \mathrm{~K})$, it should be interesting to study the behavior of $\Gamma$ at lower temperatures. In view of a close analogy in the behavior of electron acoustic absorption coefficient in superconductors and the relaxation absorption of sound in two-level systems (TLS) in the $s$-phase, one could expect a nonexponential drop in $\Gamma_{s}(T)$ in the low-temperature "tail,", or an exponential decrease but with a much smaller gap if the magnetic depairing effects are significant. It is shown in the present work that the $\Gamma_{s}(T)$ dependence at low temperatures can be described quite correctly by the tunnel model $(\mathrm{TM})^{2}$ in the framework of the standard BCS approximation, which rules out the gapless phase hypothesis. The refined value of the transition temperature for the bulk of the sample was found to be close to $T_{c}$, while the value of $T_{c m}$ determined from magnetic measurements is apparently associated with the surface phase. However, the behavior of $\Gamma$ in the vicinity of $T_{c}$ does not conform to the standard TM: the drop in $\Gamma_{s}(T)$ below $T_{c}$ begins much later than what is predicted by TM. Besides, in a certain temperature interval below $T_{c}$, the absorption is slightly higher than $\Gamma_{n}(T)$. Such effects were observed earlier in the amorphous alloy $\mathrm{Pd}_{30} \mathrm{Zr}_{70}$ and were explained qualitatively by the electron renormalization of the parameter of TLS interaction with an elastic wave. ${ }^{3}$ However, possible mechanisms of such a renormalization were not discussed and quantitative estimates of its magnitude were not obtained in Ref. 3. We shall use the mechanism of adiabatic renormalization of the amplitude of coherent tunneling ${ }^{4}$ to explain the peculiarities of sound absorption in the vicinity of $T_{c}$. This approach gives an acceptable quantitative description of the dependence $\Gamma_{s}(T)$.

The basic postulate of TM, which was confirmed irrefutably in experiments (see the review by Hunklinger and Raychaudhuri ${ }^{2}$ ), is the assumption that glasses have doublewell potentials with a tunneling link between the wells whose density of states $\bar{p}$ is constant in the space of the parameters $\xi$ and $\ln \Delta_{0}$ ( $\xi$ is the asymmetry of a double-well potential and $\Delta_{0}$ the amplitude of coherent tunneling). The response of the TLS system to an external perturbation is determined by the average over the TLS ensemble. For the sake of convenience, averaging is usually carried out by using new variables $E=\sqrt{\xi^{2}+\Delta_{0}^{2}}$ and $u=\Delta_{0} / E$ in which the density of states of TLS is independent of $E$ :

$$
g(E, U)=\frac{\bar{p}}{u \sqrt{1-u^{2}}} \equiv g(u) .
$$

Under the conditions of the experiment $(\omega \ll T, \omega$ being the frequency of acoustic vibrations), the attenuation of sound associated with the TLS is determined by the relaxation mechanism and is described by the following standard expression: ${ }^{2}$

$$
\left(\frac{\Gamma \mathrm{v}}{\omega}\right)_{\mathrm{rel}}=\int_{0}^{E_{g} / T} \frac{d \varepsilon}{\cosh ^{2}(\varepsilon / 2)} \int_{0}^{1} C_{g}(u)\left(1-u^{2}\right) \frac{\omega \nu}{\omega^{2}+\nu^{2}} d u
$$

Here $\varepsilon=E / T, E_{g} \gg T$ is the limiting energy, and $\nu$ the relaxation frequency. In Eq. (2) and below, we have used the 
system of energy units $(\hbar=k=1)$. The order of magnitude of the TLS contribution to the velocity and attenuation of sound is determined by the parameter $C$. In the standard $\mathrm{TM},{ }^{2}$ this quantity is a constant: $C=C_{0}=\bar{p} \gamma^{2} /\left(\rho \mathrm{v}^{2}\right)(\gamma$ $=1 / 2(\partial \xi / \partial e)$ is the deformation potential, $e$ the deformation, and $\rho$ the density), although a number of experimental facts can be explained only by assuming that $C$ depends on $E, u$ or $T$.

The TLS relaxation is due to their interaction with electrons as well as phonons, although the contribution of the latter can be disregarded for $T \lesssim 1 \mathrm{~K}$. The intensity of interaction of TLS with electrons is determined by the dimensionless parameter $\eta=2 n_{0}\left(V_{k k^{\prime}}^{2}\right)^{1 / 2}$, where $n_{0}$ is the density of electron states at the Fermi level and $\overline{V_{k k}^{2}}$ is the square of the matrix element of electron scattering at TLS from the state $\mathbf{k}$ to the state $\mathbf{k}^{\prime}$, averaged over the Fermi surface.

In the standard TM, the interaction of TLS with electrons is considered in the framework of the perturbation theory in parameter $\eta^{2}$, which does not change the system of energy levels. ${ }^{5}$ The entire distinction between a metallic glass and an amorphous insulator is reflected just in the emergence of a new relaxation channel with a characteristic rate

$$
\nu=\frac{\pi \eta^{2}}{2} T u^{2} J(\varepsilon) .
$$

In the $n$-phase, $J(\varepsilon)=J_{n}(\varepsilon)=(\varepsilon / 2) \operatorname{coth}(\varepsilon / 2)$ and $\nu \approx \eta^{2} T u^{2}$. As long as $\omega \ll T$, there always exist TLS with $\nu_{\mathrm{opt}} \sim \omega$, and the absorption (2) is practically independent of temperature ("plateau" region).

In the $s$-state, we must use instead of $J_{n}(\varepsilon)$ the function $J_{s}(\varepsilon, \Delta)\left(\Delta=\Delta_{s} / T, \Delta_{s}\right)$ is the superconducting energy gap: ${ }^{6}$

$$
\begin{aligned}
J_{s}(\varepsilon, \Delta)= & \frac{1}{2} \int_{\Delta}^{\infty} d \varepsilon^{\prime} \frac{f\left(-\varepsilon^{\prime}\right)}{\sqrt{\varepsilon^{\prime 2}-\Delta^{2}}}\left\{\frac{\varepsilon^{\prime}\left(\varepsilon^{\prime}-\varepsilon\right)-\Delta^{2}}{\sqrt{\left(\varepsilon^{\prime}-\varepsilon\right)^{2}-\Delta^{2}}}\right. \\
& \times \frac{f\left(\varepsilon^{\prime}-\varepsilon\right)}{f(-\varepsilon)} \Theta\left[\left(\varepsilon^{\prime}-\varepsilon\right)^{2}-\Delta^{2}\right] \operatorname{sgn}\left(\varepsilon^{\prime}-\varepsilon\right) \\
& +(\varepsilon \rightarrow-\varepsilon)\},
\end{aligned}
$$

where $f(x)$ is the Fermi function and $\Theta(x)$ is the step $\Theta$-function. The function $J_{s}(\varepsilon, \Delta)$ is frequently encountered in the theory of kinetic properties of superconductors. It has a discontinuity at $\varepsilon=2 \Delta$, while $a J_{s}(\varepsilon, \Delta) \rightarrow 2 f(\Delta)$ for $\varepsilon \ll 2 \Delta$. As a result of a rapid drop in the value of $J_{s}$ below $T_{c}$, the maximum relaxation rate $(u=1)$ becomes less than $\omega$ starting from a certain temperature while $\Gamma_{s}(T)$ "freezes."

A nonperturbative analysis ${ }^{4,7}$ revealed a more complicated pattern. Even at $T=0$, the initial coherent tunneling amplitude $\Delta_{0}$ in the $n$-phase is renormalized as the adiabatic part of the interaction of TLS with electrons is taken into consideration:

$$
\Delta_{0}^{*} \approx \Delta_{0}\left(\frac{\Delta_{0}}{\omega_{0}}\right)^{\eta^{2} / 4-\eta^{2}}
$$

where $\omega_{0}$ is the energy of the order of Debye energy.

For $T \neq 0$, the TLS ensemble can be divided arbitrarily into three intervals according their position on the $E$-scale in the $n$-state.

(1) The coherent tunneling region $E^{*} \sqrt{ } \xi^{2}+\Delta_{0}^{* 2}>T$

(2) The region $E^{*}<T<4 \widetilde{E} /\left(\pi \eta^{2}\right)$ of coherent tunneling with an amplitude $\widetilde{\Delta}=\Delta_{0} \times\left(2 \pi T / \omega_{0}\right)^{\eta^{2} / 4}$ and the energy splitting $\widetilde{E}=\sqrt{\xi^{2}+\widetilde{\Delta}^{2}}$. Going over to renormalized variables $E^{*}$ and $\widetilde{E}$ as well as to $u^{*}$ and $\widetilde{u}$ during averaging in each of the regions 1 and 2, relations (2) and (3) remain valid.

(3) The low-energy TLS $T>4 \widetilde{E} /\left(\pi \eta^{2}\right)$. In this region also, the tunneling is incoherent and has an amplitude $\widetilde{\Delta}$. However, the vanishing of the factor $\left(1-\widetilde{u}^{2}\right)$ from Eq. (2) is a reflection of the fact that the incoherent transitions between broadened levels occur with a variation of energy even in the symmetric case. The relaxation frequency

$$
\nu_{3} \approx \frac{2}{\pi \eta^{2}} T \widetilde{u}^{2} \widetilde{\varepsilon}^{2} \frac{1}{j(\widetilde{\varepsilon})}
$$

also changes in region 3.

It would appear that as a result of a decrease in $\nu_{3}$ for small $\widetilde{\varepsilon}$ [Eq. (6)], the contribution to $\Gamma$ from the part of TLS with $\widetilde{E}<\sqrt{\omega T}$ must decrease. However, this decrease is compensated by an increase in the contribution from symmetric TLS, and the partial contribution from region 3 to $\Gamma$ remains practically the same as that calculated in the standard TM. The contribution from region 2 also remains unchanged. Only the contribution from coherently tunneling TLS (region 1) undergoes significant variation. Upon a transition to the variables $E^{*}$ and $u^{*}$, the density of states $g\left(u^{*}\right)(1)$ is renormalized as a result of a nonlinear relation (5) between $\Delta_{0}^{*}$ and $\Delta_{0}$, and acquires an additional factor $\left(1-\eta^{2} / 4\right)$. The parameter $C$ is also renormalized accordingly. If the boundary between regions 1 and 2 is located at $E^{*} \sim T$, the resulting value of $\Gamma$ in the $n$-phase decreases in spite of the fact that the denominator in (2) decreases the contribution from the high-energy TLS. Below $T_{c}$, the nonlinear relation (5) is rapidly transformed into a linear relation: ${ }^{4} \Delta_{0}^{*}$ $\approx \Delta_{0}\left(\Delta_{s} / \omega_{0}\right)^{\eta^{2} / 4}$, renormalization of $C$ vanishes, and $\Gamma_{s}(T)$ below $T_{c}$ may increase before "freezing out."

Let us now discuss the experimental results. Figure 1 shows the dependence $\Gamma_{s}(T) / \Gamma_{n}\left(T_{c}\right)$ for transverse sound. The normalization factor $\Gamma_{n}\left(T_{c}\right)^{-1}$ used for presenting the results can be easily determined from the variation of the amplitude of the acoustic signal between $T_{c}$ and the deep superconducting state.

In the region of the low-temperature "tail," the renormalization $g(u)$ can be disregarded. The following estimate is obtained from (1), (2) and (4):

$$
\frac{\Gamma_{s}(T)}{\Gamma_{n}\left(T_{c}\right)}=\frac{2 \pi \eta^{2}}{3 \omega} T \mathrm{e}^{-\Delta_{s} / T}, \quad\left(T / T_{c}<0.3\right) .
$$

According to this equation, the low-temperature region $\Gamma_{s}(T)$ must become linear in coordinates $\ln \left(\Gamma_{s}(T) T^{-1}\right), T^{-1}$. 


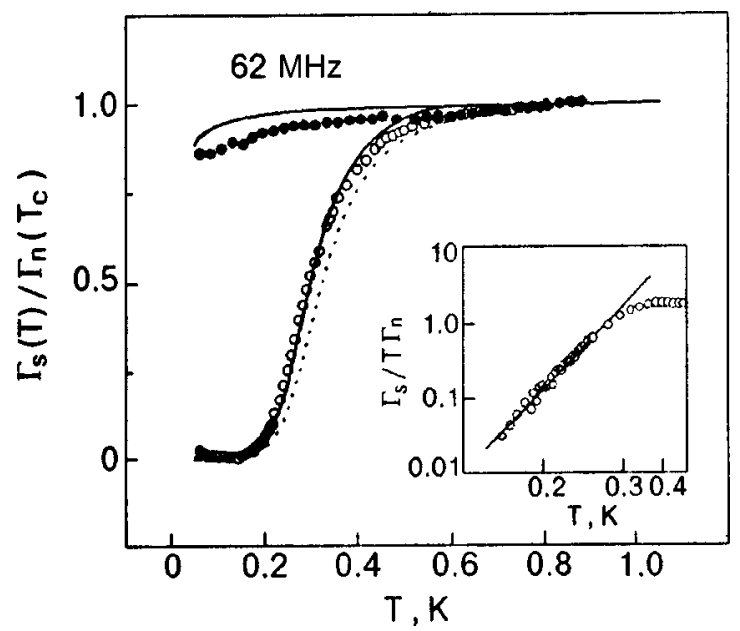

FIG. 1. Temperature dependence of attenuation in $n(\bullet)$ and $s(\bigcirc)$ states. The solid curves correspond to calculations for $\eta=0.65$ and $T_{c}=0.83 \mathrm{~K}$ and the dotted ones to $T_{c}=0.9 \mathrm{~K}$. The inset illustrates the evaluation of $\Delta_{s}(0)$ and $\eta$. The solid line corresponds to the linear approximation.

as can be seen from the inset to Fig. 1. The slope of the approximating straight line is determined by the superconducting energy gap which is in good accord with the BCS value: $\Delta_{s}(0) / T_{c}=1.7 \pm 0.1\left(T_{c}=0.9 \mathrm{~K}\right)$ or $\Delta_{s}(0) T_{c}=1.8$ $\pm 0.1\left(T_{c}=0.83 \mathrm{~K}\right)$. Intersection of the approximating straight line with the ordinate axis leads to the estimate $\eta$ $=0.65 \pm 0.05$. These data can also be used to refine the value of $T_{c}$ for the bulk of the sample. The slope of the approximating straight line and its interaction with the ordinate axis (see inset to Fig. 1) are by no means connected with the choice of $T_{c}$. For a complete evaluation of $\Gamma_{s}(T)$, we must use the specific value of $T_{c}$. It can be seen from Fig. 1 that the theoretical curve for $T_{c}=0.83 \mathrm{~K}$ is in much better accord with the experimental data.

The closeness of $\Delta_{s}(0) / T_{c}$ to the BCS value indicates that peculiarities in the behavior of $\Gamma(T)$ in the vicinity of $T_{c}$ are not connected with the magnetic depairing effects. Let us now discuss the applicability of the hypothesis of the electron renormalization of $C$ to the description of the behavior of $\Gamma_{s}(T)$.

Figure 2 shows the data on variation of $\Gamma_{s}(T)$ and $\Gamma_{n}(T)$ in the vicinity of $T_{c}$, measured with a higher resolution than in Fig. 1. The results are normalized to the value $C_{0}=2.85$ $\cdot 10^{-5}$ obtained from the slope of $\mathrm{v}_{s}(\ln T)$ in the deep superconducting state $(T \leqq 0.3 \mathrm{~K}) .{ }^{1)}$ The normalized value of $\Gamma\left(T_{c}\right)$ in the standard TM must be close to 0.5 for $\omega \ll T$. Renormalization (decrease) of $C$ naturally shifts $\Gamma$ towards lower values. However, it has not been possible to measure the value of attenuation with an accuracy better than $1 \%$, which would allow an analysis of the shift of the experimental dependence relative to the theoretical one. Hence we consider only relative position of the lines $\Gamma_{s}(T)$ and $\Gamma_{n}(T)$ (the latter value is obtained in a magnetic field $H \approx 2 \mathrm{~T}$ ) which could be measured with a much higher degree of accuracy (Fig. 2).

The meaning of the "anomaly" in $\Gamma$ discussed above can be seen clearly from a comparison of the experimental dependences with the theoretical ones obtained in the stan-

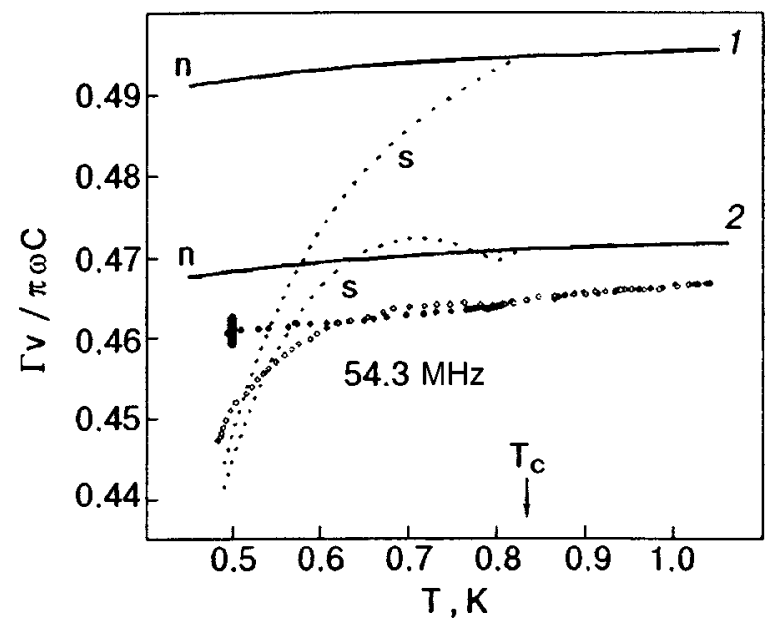

FIG. 2. Behavior of attenuation in the vicinity of $T_{c}$ in $n(\bigcirc)$ and $s(\bigcirc)$ states. The experimental recordings are averaged, and the noise level is indicated by the bold vertical line. Curves 1 and 2 are obtained without and with renormalization respectively; $\eta=0.65, d=1.2$.

dard TM (curve 1 in Fig. 2). According to calculations, a decrease in absorption begins at $T_{c}$ and continues as the steepness increases upon a decrease in temperature. The experimental dependence clearly displays a different behavior: $\Gamma_{s}(T)$ does not display any variation at $T_{c}$ within the limits of resolution, and a tendency towards an increase in $\Gamma_{s}(T)$ over $\Gamma_{n}(T)$ is observed at lower temperatures. In any case, $\Gamma_{s}(T)$ remains practically unchanged over a wide temperature range $T<T_{c}$. More clearly manifested effects of this kind were detected earlier in the alloy $\mathrm{Pd}_{30} \mathrm{Zr}_{70} \cdot{ }^{3}$ It was also suggested in Ref. 3 that these anomalies can be associated with the electron renormalization of the parameter $C$.

The renormalization of $C$ indeed takes place in the alloy investigated by us. An irrefutable proof of this is the intersection the dependences $\mathrm{V}_{s}(T)$ and $\mathrm{v}_{n}(T)$ at a quite low temperature $T_{\text {cr }}$ (its value for $62 \mathrm{MHz}$ is $T_{\mathrm{cr}}=0.055 \mathrm{~K}$ ). The scale of renormalization is quite significant $\left(\delta C / C_{0} \sim 0.25\right)$ and is about double the quantity $\eta^{2} / 4 \sim 0.09-0.12$, which allows us to assume the existence of several mechanisms of renormalization. ${ }^{2)}$ Moreover, incompatibility of the scale of $\delta C / C_{0}$ with the anomalies in $\Gamma_{s}(T)$ indicates that these mechanisms affect only insignificantly the TLS forming the relaxation attenuation for $T \sim T_{c}$. It should be recalled that the main contribution to $\Gamma(T)$ comes from asymmetric TLS with $u_{\mathrm{opt}} \sim \sqrt{\omega / T} \ll 1$. One possible mechanism of renormalization, which takes into account the fluctuational rearrangement of the barrier in a double-well potential, is associated only with the symmetric $\mathrm{TLS}^{8}$ and apparently makes no contribution to $\Gamma(T)$.

The adiabatic renormalization does not impose any constraints on the possible values of $u{ }^{7}$ In spite of the fact that the condition $\varepsilon>1$ moves the coherently tunneling TLS to the region of action of the truncating factor in (2), their partial contribution to $\Gamma(T)$ may be quite significant on the scale of Fig. 2.

For the purpose of numerical computations, we used the model energy dependence of the renormalization parameter 


$$
\frac{C}{C_{0}}=1-\Theta(\varepsilon-d)[1+(2 f(\Delta)-1) \Theta(2 \Delta-\varepsilon)] \frac{\eta^{2}}{4},
$$

where $d$ is a free fitting parameter. The first cofactor in the term describing renormalization in Eq. (8) defines the boundary between the regions 1 and 2 . Since the coherent amplitude $\Delta_{0}^{*}$ decreases exponentially for $\varepsilon<1,{ }^{4}$ such an approximation seems to be quite reasonable. The second cofactor in (8) takes into account the fact that only normal excitations can contribute to renormalization for $\varepsilon<2 \Delta$.

The results of calculations are also presented in Fig. 2 (curves 2). The interval of approximate "independence" of $\Gamma_{s}(T)$ can be matched with that observed for a given value $\eta=0.65$ for a quite reasonable value of $d=1.2 \pm 0.1$. It can be seen that the calculated dependence $\Gamma_{s}(T)$ varies initially below $T_{c}$ in the same manner as in the standard TM. Subsequently, $\Gamma_{s}(T)$ displays a kink with a reversal of the sign of $d \Gamma / d T$ at $T=2 \Delta_{s} / d$. The emergence of the kink is a consequence of the use of a step approximation in (8). As long as $2 \Delta_{s}$ does not exceed the value of $E=T d$, superconductivity does not have any effect on renormalization. Apparently, the restriction on the renormalization of $C$ imposed from below by a smooth function of energy decreases the variation of $\Gamma_{s}(T)$ in the vicinity of $T_{c}$ and eliminates the kink. The same result is also arrived at by the broadening of the superconducting transition which is quite natural for an amorphous sample. Hence it can be really expected that $\Gamma_{s}(T)$ will not change at $T_{c}$, as is indeed observed in the experiments.

Thus, the evolution of $\Gamma_{s}(T)$ near $T_{c}$ is determined by two factors, viz., a drop in $\Gamma_{s}(T)$ due to a decrease in the relaxation rate $\nu$, and an increase in $\Gamma_{s}(T)$ as a result of "freezing out" of the renormalization of $C$. In contrast to the latter factor, the former is frequency-dependent, and hence the resulting variation of $\Gamma_{s}$ will also depend on frequency. Upon a decrease in $\omega$, the temperature interval in which $\Gamma_{s}(T)>\Gamma_{n}(T)$ must expand, and vice versa. In particular, calculations show that an increase in frequency by an order of magnitude (measurements were made just at these frequencies by Esquinazi $e t a l .^{3}$ ) completely masks the effect of the second factor for the same values of $\eta, T_{c}$, and $d$. However, Esquinazi et al. ${ }^{3}$ carried out measurements on glass with $T_{c} \simeq 2.5 \mathrm{~K}$. In this region, $\nu$ is determined mainly by phonons and depends weakly on the state of the electron subsystem. Under these conditions, the "freezing out" of renormalization must give an even more pronounced effect than in our experiments, as was apparently observed by Esquinazi et al. ${ }^{3}$
In conclusion, let us formulate the main results. The experimental dependence of the absorption of sound in the amorphous superconducting alloy $\mathrm{Zr}_{41.2} \mathrm{Ti}_{13.8} \mathrm{Cu}_{12.5} \mathrm{Ni}_{10} \mathrm{Be}_{22.5}$ was used to determine the superconducting energy gap (which is found to be practically identical to the gap obtained in the BCS theory) and the parameter $\eta$ characterizing the intensity of interaction of TLS with electrons. The departures from the predictions of the standard tunneling model observed in the vicinity of $T_{c}$ can be explained qualitatively and quantitatively by the adiabatic renormalization of the coherent tunneling amplitude.

The authors are obliged to Prof. G. Weiss for drawing their attention to the publications by Kagan and Prokof'ev ${ }^{4}$ and Stockburger et $_{\text {al. }}{ }^{7}$

This research was partially supported by the State Foundation on Fundamental Research in Ukraine (grant No. 2.4/ 153) and by Deutsche Forschungsgemeinschaft via SFB 252. One of the authors (W. L. J.) wishes to thank the US Energy Department for grant (No. DE-FG03-86ER45242), while S. V. Zh. is grateful to the von Humboldt Foundation for financial support.

\footnotetext{
*E-mail: fil@ilt.kharkov.ua

${ }^{1)}$ This value of $C_{0}$ is double the analogous value presented in Ref. 1 . The departure is due to the fact that the value of $C_{0}$ was estimated in Ref. 1 by using the linear dependence $\mathrm{v}_{n}(\ln T)$ whose slope depends significantly on the renormalization of $C$. The latter was not taken into consideration in Ref. 1. The value of $\eta$ presented in Ref. 1 is also found to be exaggerated for the same reason.

${ }^{2)}$ The effect of renormalization of $C$ on the velocity of sound will be considered in a separate publication.
}

\footnotetext{
${ }^{1}$ A. L. Gaiduk, E. V. Bezuglyi, V. D. Fil, and W. L. Johnson, Fiz. Nizk. Temp. 23, 1139 (1997) [Low Temp. Phys. 23, 857 (1997)].

${ }^{2}$ S. Hunklinger and A. K. Raychaudhuri, in Prog. in Low Temp. Phys. (Ed. by D. F. Brewer), Vol. 9, North-Holland, Amsterdam (1986).

${ }^{3}$ P. Esquinazi, H.-M. Ritter, H. Neckel et al., Z. Phys. B: Condens. Matter 64, 81 (1986).

${ }^{4}$ Yu. Kagan and N. V. Prokof'ev, Zh. Éksp. Teor. Fiz. 97, 1698 (1990) [Sov. Phys. JETP 70, 957 (1990)].

${ }^{5}$ J. L. Black, B. L. Gyorffy, and J. Jäckle, Philos. Mag. B 40, 331 (1979).

${ }^{6}$ J. L. Black and P. Fulde, Phys. Rev. Lett. 43, 453 (1979).

${ }^{7}$ J. Stockburger, U. Weiss, and R. Görlich, Z. Phys. B: Condens. Matter 84, 457 (1991).

${ }^{8}$ K. Vladar and A. Zawadowski, Phys. Rev. B 28, 1564, 1582, 1696 (1983).
}

Translated by R. S. Wadhwa 